\title{
PREVISÃO DO CONSUMO DE ALUMÍNIO PRIMÁRIO NO BRASIL POR MEIO DE MODELOS DE INTENSIDADE DE USO
}

\author{
SAUL B. SUSLICK*
}

\begin{abstract}
FORECASTING OF PRIMARY ALUMINIUM CONSUMPTION IN BRAZIL BY INTENSITY OF THE USE MODELS. Several new and improved long-range forecasting techniques for mineral demand projection for aluminium consumption in Brazil are presented. The proposed methods were specified within the framework of the intensity of use concept (ratio of the quantity of a mineral commodity consumed in a nation to its gross domestic product). An evaluation procedure was used to estimate the behavior of each method in relation to different criteria: accuracy, cost, degree of complexity, sensitivity of a technique to changes in technology and material substitution and requirements (e.g. availability of historical data, development-time, manipulation, utilization by people not acquainted with quantitative techniques in the mineral industry). It is concluded that there is not an all-purpose technique which fui out all requirements for long-range demand forecasting. Another major finding of this paper is that learning model and vector projections models (time varying coefficients) were very useful in forecasting in the expost period (1981-87) by incorporating several variables such as own price, substitute prices, technical change and others in the dynamics of mineral demand. Brazil's aluminium consumption in the year 2000 is forecasted to be in the range of 800,000 to $1,300,000$ metric tons; these bounds correspond to rates of growth of GDP of $3.6 \%$ and $6.1 \%$, respectively. This range covers the forecasts made by the extensive translog consumption model and time varying coefficients model, both of which gave high accuracies in the expost forecast period.
\end{abstract}

Keywords: Intensity of use, mineral consumption, forecasting methods.

\begin{abstract}
RESUMO Neste trabalho são apresentadas novas técnicas de previsão da demanda mineral de longo prazo aplicadas na projeção do consumo de alumínio primário no Brasil para o ano 2000. Os métodos propostos foram definidos utilizando-se o conceito da intensidade de uso (razão do consumo mineral por unidade de PIB). Como critério de avaliação, foram utilizados exatidão, custo, grau de complexidade, sensibilidade às mudanças tecnológicas e substituição e requisitos básicos (disponibilidade de séries históricas, tempo de desenvolvimento, manipulação e uso por indivíduos não familiarizados com técnicas quantitativas da industria mineral). Pode-se concluir que não existe uma técnica que isoladamente contemple todos os requisitos mencionados para a previsão mineral. Os modelos de projeções vetoriais (coeficientes temporais) e conhecimento (learning systems) forneceram bons ajustes para o período-teste (1981-1987), por incorporar variáveis como preço do metal, preço do metal-substituto, mudança tecnológica e demais parâmetros da demanda mineral. O consumo de alumínio no ano 2.000 foi estimado entre 800.000 e 1.300 .000 toneladas, correspondendo estes limites a taxas anuais de crescimento do PIB de $3,6 \%$ e $6,1 \%$ respectivamente, que são abrangidos pelo modelo translog.
\end{abstract}

Palavras-chaves: Intensidade de uso, consumo mineral, métodos de previsão.

INTRODUÇÃO A indústria mineral já vem há longo tempo, utilizando os métodos de previsão da demanda na gestão de suas atividades. Durante os anos 50 e 60 , as previsões ainda podiam ser realizadas por simples projeções com certo grau de confiabilidade devido ao crescimento econômico constante e baixas taxas inflacionárias. Entretanto, a crise de energia em 1973 e as sucessivas mudanças do cenário internacional adicionaram uma dose crescente de incerteza e complexidade aos eventos, indicando a necessidade de métodos mais robustos e abrangentes.

A previsão do consumo de uma substância mineral constitui um elemento importante no processo de decisão no setor mineral na medida em que possibilita simular os impactos das substituições, as tendências das mudanças tecnológicas e a direção dos padrões de consumo mineral. Tendo em vista as complexas relações entre a oferta e a demanda dos produtos minerais e a dificuldade de acompanhamento atual e sistemático do mercado mineral, optou-se por um tratamento da previsão a longo prazo.

O objetivo deste trabalho é realizar a previsão do consumo de alumínio no ano 2.000 no Brasil, utilizando técnicas inéditas e que incorporem as complexas relações entre o consumo de alumínio, preço e seus principais determinantes. A técnica da intensidade de uso (função que mede a razão entre a quantidade consumida da substância mineral e a renda per capita) será utilizada para descrever a demanda, tanto na sua forma original conhecida por Malenbaum (1975), como em modelos com maior complexidade que busquem capturar todas as especificidades das variáveis econômicas no consumo mineral.

Serão apresentadas novas técnicas, como as curvas de conhecimento (learning curves) empregadas inicialmente no planejamento estratégico industrial e na área mercadológica e de produção. As curvas de conhecimento ou de aprendizado são pouco conhecidas na área mineral e podem representar uma grande contribuição na previsão da demanda e da mudança tecnológica, pela sua simplicidade e operacionalidade.

Uma alternativa mais formal porém mais complexa que a intensidade de uso e as curvas de conhecimento, são os modelos fatoriais que serão abordados brevemente neste trabalho, que empregam os escores fatoriais e coeficientes temporais combinados em complexas funções para capturar a dinâmica da demanda mineral.

A parte final do trabalho abrange uma discussão dos métodos apresentados, não somente sobre sua exatidão, mas também cotejando as vantagens e desvantagens de cada um, envolvendo custo, grau de complexidade, mudança tecnológica, requisitos e facilidade de manuseio.

CONSIDERACÕES BÁSICAS SOBRE A PREVISÃO DA DEMANDA MINERAL Existe uma considerável ambigüidade sobre o significado do termo "demanda" na área mineral. Ele não representa somente as necessidades em 
termos absolutos e nem tampouco consumo, mas sim as diferentes quantidades de uma substância mineral que os consumidores gostariam de adquirir sob diferentes condições de preço e renda. Quando se realiza a previsão da demanda mineral, deve-se considerar que o passado é um amálgama das forças da oferta e da demanda atuando conjuntamente. Neste trabalho, em algumas projeções da demanda futura, assume-se também que estejam relacionadas a eventos que ocorreram com a oferta em passado recente.

A previsão do consumo e bastante complexa de formular dentro da análise oferta-demanda. Os métodos variam muito e não existe uma forma única de abordagem, conforme assinala Suslick (1990). Estes devem abranger os fatores que desempenham papel-chave como determinantes nos estudos de demanda: o nível da atividade econômica, o preço real do metal ou commodity a ser analisado, o preço real dos substitutos e, por fim, as mudanças tecnológicas, conforme mostra a figura 1 . Nas análises dos determinantes do consumo mineral a longo prazo devem-se considerar também as variações na composição do PIB (Produto Interno Bruto), assim como as suas composições, segundo preconizam Roberts (1985) e Suslick \& Harris (1991).

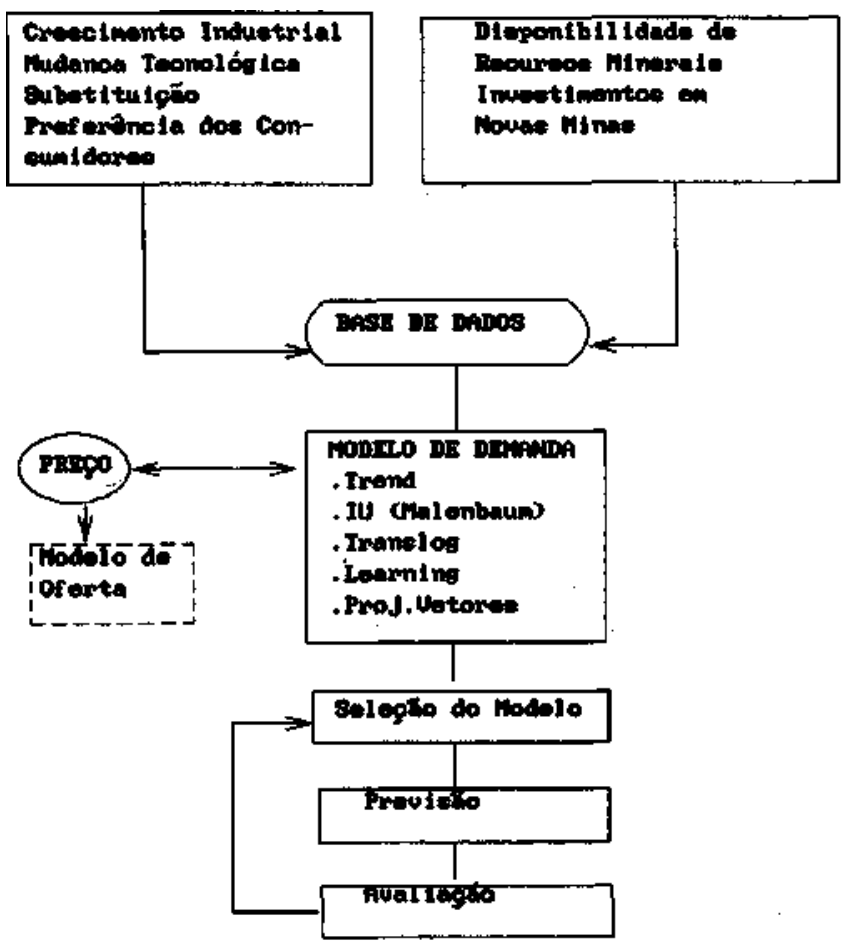

Figura 1 - Metodologia utilizada na previsão da demanda mineral

Figure 1 - Methodology used for mineral demand forecasting

Quando o objetivo é a previsão do consumo mineral a longo prazo, o uso indiscriminado de modelos econométricos mais complexos e abrangentes, no que concerne às variáveis econômicas, nem sempre é satisfatório, quando comparado com modelos mais simples baseados na projeção de tendências, modelos de ciclos de vida e de conhecimento, embora existam desvantagens quando a previsão é unicamente baseada na análise de padrões das projeções, onde os resultados nem sempre apresentam um sentido econômico. Este fato se deve à presença de pontos de inflexão no consumo, pela mudança da estrutura econômica no futuro, que não é detectada quando se realizam as projeções. Um extenso trabalho sobre os diversos métodos de projeção de séries temporais pode ser encontrado em Gregg et al(1968), uma adaptação para a previsão da demanda mineral de diferentes substancias nos EUA foi elaborada por Harris \& Jeon (1987) e na previsão do consumo de alumínio no Brasil por Suslick (1990).

BREVES CONSIDERAÇÕES SOBRE O ALUMÍNIO NO BRASIL O Brasil tornou-se auto-suficiente em alumínio no início da década de 80 (Tab. 1) pela combinação de um imenso potencial hidroelétrico e de grandes reservas minerais de bauxita. Embora o país possua um grande mercado interno para o consumo do metal, cerca de metade de sua produção é exportada, situando o Brasil entre os dez maiores exportadores de alumínio do mundo. Os projetos de expansão ALUMAR e ALBRÁS permitiram ao país aumentar a sua produção, atingindo 1,25 milhões de toneladas em 1995.

Segundo Braz-Pereira (1988), existem duas regiões produtoras de alumínio no país que apresentam diferentes con-

Tabela 1 - Oferta e demanda de alumínio primário no Brasil Table 1 - Supply and demand of primary aluminium in Brazil (in thousand of tonnes)

\begin{tabular}{|c|c|c|c|c|c|c|c|c|c|}
\hline & 1979 & 1980 & 1981 & 1962 & 1963 & 1994 & 1985 & $19: 6$ & 1987 \\
\hline Productio & 238 & 261 & 256 & 299 & 401 & 455 & 549 & 757 & 844 \\
\hline Rearpentugto Scound. & s & 50 & 42 & 43 & 45 & 4 & 52 & 58 & 61 \\
\hline Impintacklp & 79 & 69 & 37 & 15 & 6 & 10 & 7 & 7 & 14 \\
\hline Exporthgato & 9 & 12 & 21 & 18 & 170 & 2005 & 215 & 357 & 463 \\
\hline Conseno (I) & 257 & 285 & 262 & 282 & $27 !$ & 205 & 347 & 424 & 430 \\
\hline $\begin{array}{l}\text { Consmo per eapita } \\
(\mathbf{k}+\mathrm{H}+\mathrm{b})\end{array}$ & 3,0 & $\mathbf{3}, \mathbf{0}$ & 2,4 & 2,5 & 2,2 & 2,2 & 2.6 & 3,1 & 29 \\
\hline
\end{tabular}

textos econômicos. A região mais antiga, situada no sul do pais é voltada, em grande parte, para o mercado interno, e a região norte (Amazônia), que possui grande potencial hidroelétrico e goza de enormes incentivos e subsídios governamentais, para assegurar a sua competitividade no mercado internacional.

O consumo de alumínio no Brasil cresceu de maneira exponential de 1962 a 1974, diminuindo em seguida e atingindo um patamar durante o início dos anos 80, mas exibiu uma pequena retomada em 1984 e 1985. O padrão errático da IU (intensidade de uso) e do consumo resulta de diferentes fatores, destacando-se a influência dos choques do petróleo no crescimento econômico, combinada com medidas macroeconômicas e de políticas de preços durante as últimas décadas.

O MQDELO DE INTENSIDADE DE USO E AS IMPLICAÇOES ECONÔMICAS Um dos estudos mais interessantes sobre a análise da demanda mineral é a intensidade de uso (IU), proposta por Malenbaum $(1973,1975,1978)$, que define a razão entre a quantidade da substância mineral consumida $\left(D_{t}\right)$ e a renda nacional $\left(Y_{t}\right)$, expressa tanto na forma de PIB ou PNB. Malenbaum mostrou que a relação de IU em função da renda per capita, y, exibe a forma de um "U" invertido (assimetria negativa) para economias altamente industrializadas: $\mathrm{IU}=f(\mathrm{y})$, onde o padrão de y em relação a IU aproxima-se da lognormal.

A lógica da hipótese de Malenbaum está ligada aos padrões de uso das substâncias minerais em função do ciclo de desenvolvimento de uma nação. Nos primeiros estágios de crescimento econômico, os requisitos para utilização das matérias-primas são muito reduzidos e, por conseqüência, o seu consumo é elevado, tendo em vista que a economia se encontra mergulhada numa fase ainda pré-industrial. A partir 
do aumento de renda e da expansão do processo de industrialização, o consumo de metais diminui quando são atendidas as necessidades básicas e de infra-estrutura. Nesse período econômico, a tendência do consumo aumenta gradativamente em direção ao setor de serviços. Toda esta lógica induziu Malenbaum (1975) a propor a lei da demanda mineral.

Embora a técnica de IU seja bastante simples e fácil de aplicar, essa lei da demanda foi muito criticada pela economia mineral. A principal restrição é que o modelo de IU, na forma proposta por Malenbaum, não considera o uso dos materiais numa economia de maneira abrangente, por não contabilizar o material contido nas importações e exportações. Sendo os dados de consumo aparentes, a IU é uma simples medida do uso da matéria-prima. Se o país é um grande importador e/ou exportador de produtos que contêm a referida matéria-prima, a medida da IU subestima e/ou superestima a IU real dentro da economia.

Uma análise parcial desses efeitos no consumo de cobre nos EUA foi realizada por Hutchison \& Tilton (1987), e na demanda de enxofre nos EUA por Alrawhi \& Rieber (1990).

Uma segunda crítica ao modelo de Malenbaum é a de ignorar o efeito dos preços na substituição dos materiais, e da tecnologia na economia dos materiais e na inovação dos produtos, por descrever IU somente como função da renda (y). Segundo Canavan (1983), Roberts (1985) e Considine (1991), as mudanças técnicas, substituição de materiais e preferências dos consumidores possuem fortes impactos na IU. Tilton (1985) desenvolveu uma estrutura econômica ao decompor RJ em duas componentes: PCI e MCP, onde o PCI é a composição do produto da renda e MCP é a composição material do produto, definida pela seguinte expressão:

$\mathrm{IU}=\mathrm{PCI} \times \mathrm{MCP}=(\mathrm{Q} / \mathrm{Y}) \times(\mathrm{D} / \mathrm{Q})$,

onde:

$\mathrm{Q}=$ conteúdo da matéria-prima nos produtos

De modo geral, PCI é fortemente influenciado pelas preferências dos consumidoes e demais determinantes da demanda dos bens duráveis, enquanto o MCP é determinado pela tecnologia de produção e seus efeitos na demanda derivada dos materiais e pelos preços relativos dos substitutos. Roberts (1988), analisando diversos metais nos EUA, estimou que as mudanças na IU provocadas pelo PCI possuem magnitude maior que a do MCP. Resultados semelhantes foram encontrados na análise do consumo de metais no Brasil por Sulslick $\&$ Harris (1990).

Harris \& Jeon (1987), numa tentativa de preservar a robustez do padrão lognormal inicialmente proposto por Malenbaum e ao mesmo tempo adequando-o à teoria econômica, demonstraram que a IU implica num modelo de demanda de renda na qual a elasticidade da renda da demanda varia em função do nível de renda. Esta demonstração é baseada na equivalência entre os modelos lognormal e translog:

$I U_{\mathrm{l}}=\frac{\mathrm{K}}{\sigma \sqrt{2 \pi}} \mathrm{e}-1 / 2\left(\frac{\ln y_{\mathrm{t}}-\mu}{\sigma}\right)^{2} \Rightarrow I U_{1}=A y^{\beta_{2}+\beta_{1} b y_{1}}$

onde:

$\mathrm{K}$, $\sigma$ e $\mu$ são parâmetros da curva

$\mathrm{A}, \beta_{0}$ e $\beta_{1}$, parâmetros da função translog. ${ }^{(1)}$

Partindo da definição de IU, temos que:

$\mathrm{IU}_{\mathrm{t}}=\frac{\mathrm{D}_{\mathrm{V}} / \mathrm{POP}_{\mathrm{t}}}{\mathrm{Y}_{\mathrm{V}} / \mathrm{POP}_{\mathrm{t}}}=\frac{\mathrm{d}_{\mathrm{t}}}{\mathrm{y}_{\mathrm{t}}}=\frac{\mathrm{D}_{\mathrm{t}}}{\mathrm{Y}_{\mathrm{t}}}$,

$\mathrm{d}_{\mathrm{i}}=\mathrm{IU}_{\mathrm{i}} \cdot \mathrm{y}_{\mathrm{l}}$

(1) $\begin{aligned} & \ln A=\ln K_{2}-\ln \sqrt{2} \pi-\mu^{2} / \sigma^{2} \\ & \beta_{0}=\mu / 2 \sigma^{2} \\ & \beta_{1}=-1 / 2 \sigma^{2}\end{aligned}$ onde, $d_{t}$ e $y_{t}$ são o consumo per capita e a renda per capita respectivamente, têm-se, então:

$$
d_{4}=A y^{\beta_{1}+1+\beta_{1} \ln y_{2}} \text { e } \mathrm{UU}_{1}=A y^{\beta_{0}+\beta_{1} \ln y_{t}}
$$

Finalmente: $\mathrm{D}_{\mathrm{l}}=\mathrm{d}_{\mathrm{l}} \mathrm{POP}=\mathrm{IU}_{\mathrm{l}} . \mathrm{Y}_{\mathrm{t}}$

Desse modo, a elasticidade da renda da demanda per capita é $\beta_{0}+1+\beta_{1} 1 \mathrm{ny}_{\mathrm{t}}$ e a elasticidade de renda da intensidade de uso (IU) é $\beta_{0}+\beta_{1} \operatorname{lny}_{\mathrm{t}}$. O significado desta demonstração é a formalização do vínculo entre IU e a demanda. Definindo o modelo translog por $\mathrm{T}(\mathrm{y} ; \beta)$, onde $\beta^{\prime}=\left[\mathrm{A}, \beta_{1}\right.$, $\beta_{2}$, e recorrendo-se à teoria da demanda derivada, que postula uma descrição mais abrangente da IU, na qual participam o preço da commodity $\mathrm{P}$, o preço do substituto PS e a mudança técnica $\mathrm{T}$, teremos o modelo abaixo:

$\mathrm{IU}_{\mathrm{t}}=\mathrm{T}(\mathrm{y}, \mathrm{P}, \mathrm{Ps}, \tau ; \Omega)$,

onde $\Omega$ é 0 vetor dos coeficientes do modelo translog expandido.

Dada a dificuldade de obtenção de informações abrangentes dos usos setoriais e finais para determinar as quantidades envolvidas nos produtos que contêm alumínio nas importacões e exportações brasileiras, serão utilizados ps dados de IU baseados no consumo aparente de alumínio primário. Essa simplificação baseia-se no pressuposto de que as importações e exportações oscilaram segundo um padrão histórico conhecido e determinado.

A previsão do consumo mineral, segundo os diferentes modelos de IU, são apresentados a seguir. Os valores do consumo são calculados com base na projeção do PIB segundo um modelo de tendência exponential e, posteriormente, o consumo estimado é calculado multiplicando-se a IU estimada por meio dos modelos pelo PIB estimado.

MODELO SIMPLES DE INTENSIDADE DE USO

Segundo Malenbaum (1973), a intensidade de uso (IU) pode ser descrita como função do PIB per capita (yt) da seguinte

$\mathrm{IU}_{4}=\frac{\mathrm{D}_{1}}{\mathrm{GDP}_{1}}=f\left(\mathrm{y}_{\mathrm{t}}\right)$

forma:

Dois tipos de especificações podem ser utilizados parayfyt): linea e lognormal. O modelo mais empregado para IU e y nas economias dos países industrializados é o lognormal (Eq. 1). Nesse sentido, o modelo lognormal foi ajustado aos dados de IU e de PIB per capita no Brasil, onde foi obtida a seguinte

$\mathrm{IU} \mathrm{U}_{\mathrm{t}}=\left[\frac{\mathrm{e}^{-58,5603}}{1,0962 \sqrt{2 \pi}} \mathrm{e}^{-\mathrm{lt} t}\left(\frac{\ln \mathrm{ln}_{\mathrm{t}}-3,5046}{1,0962}\right)\right]^{2} \quad \mathrm{R}^{2}=0,90$

equação após o emprego de mínimos quadrados e de algumas transformações:

A figura 2A exibe o ajuste por meio desse modelo clássico de Malenbaum para a IU, enquanto a figura $2 \mathrm{~B}$ mostra as estimativas para o consumo de alumínio primário.

Com base num simples modelo linear da forma $\mathrm{IU}=$ ao $+\alpha_{1} \mathrm{y}_{\mathrm{t}}$, e de acordo com as premissas de Malenbaum do padrão lognormal para economias relativamente maduras, pode-se obter um modelo linear de PIB para o consumo de alumínio (Fig. 3B) a partir da seguinte expressão de IU (Fig. $3 \mathrm{~A}): \mathrm{IUt}=0,2893+4,224 \times 10^{-0} \mathrm{GDP}_{\mathrm{t}}\left(\mathrm{R}^{2}=0,799\right)$. 
Fine
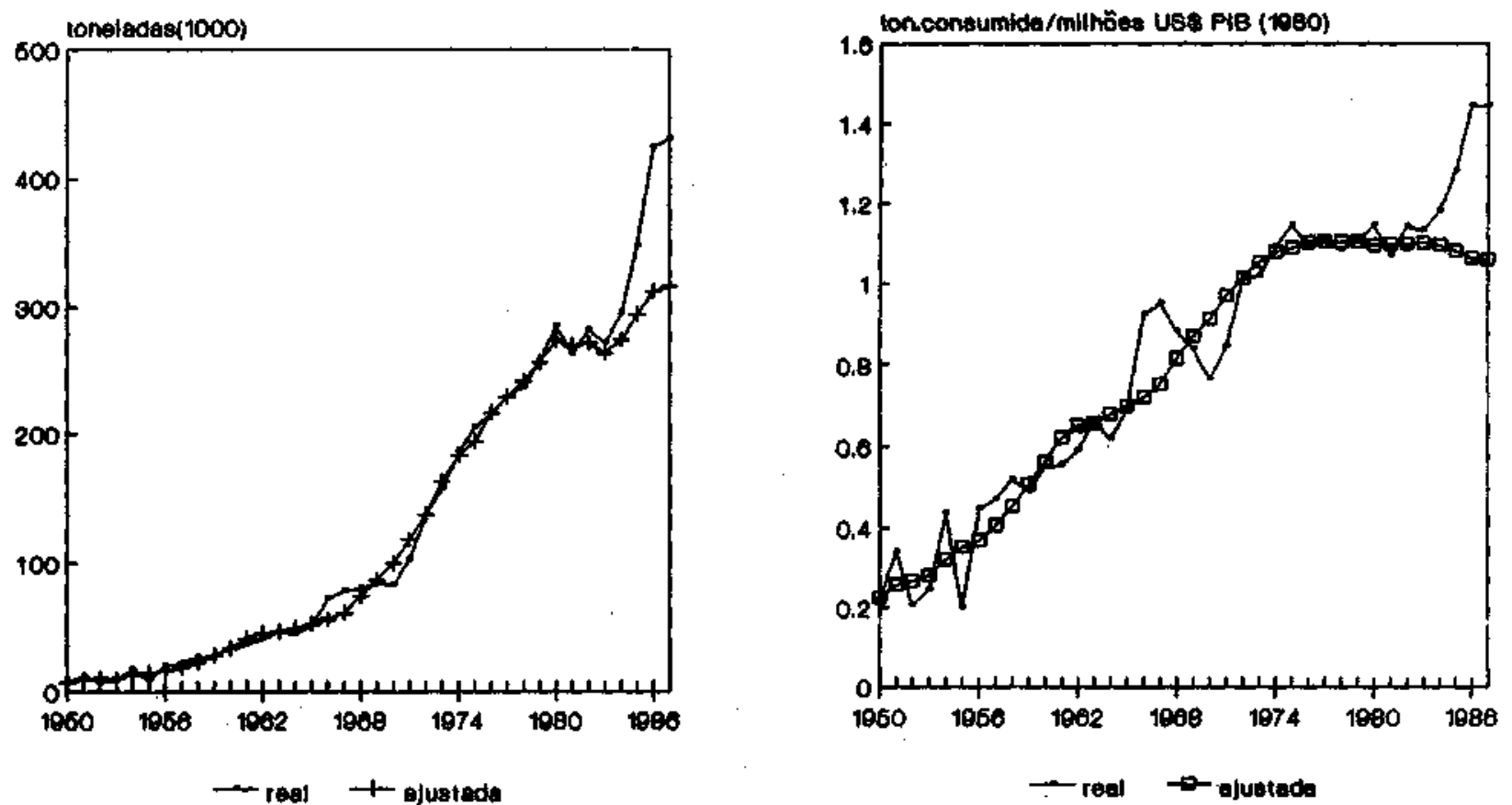

Figura 2 - Comparação entre os valores reais e estimados de intensidade de uso e consumo de alumínio baseados na $W$ como modelo lognormal do PIB per capita. A. Valores reais e estimados de intensidade de uso; B. Valores reais e estimados de consumo. Fonte: consumo de alumínio primário - Metallgesellschaft (vários números); PIB = valores constantes (1980) expressos em milhões de dólares americanos; Economic Survey of Latin America and Caribbean. ECLA/UN (vários); Statistical Yearbook for Latin America. ECLA/UN (vários); IMF (1988); IU = expresso em toneladas/milhões de dólares americano PIB (1980) Figure 2 - Comparing actual and estimated aluminium consumption and intensity of use based upon IU as a lognormal model of per capita GDP. A. Actual and fitted values for intensity of use: B. Actual and fitted values for consumption

Fig.3
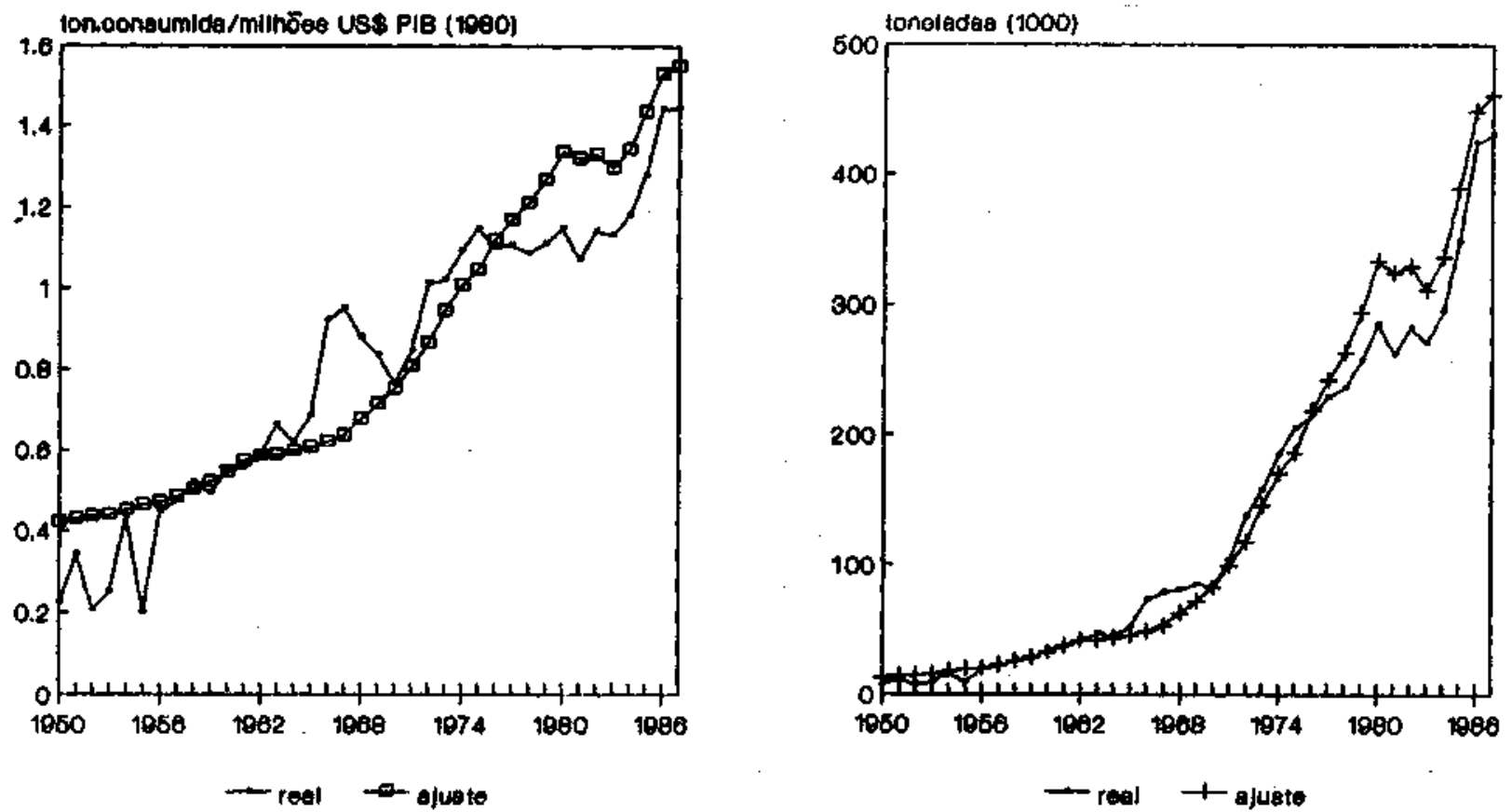

Figura 3 - Comparação entre os valores reais e estimados de intensidade de uso e consum,o de alumínio baseado na IU como modelo linear do PIB per capita. A. Valores reais e estimados de intensidade de uso; $\boldsymbol{B}$. Valores reais e estimados de consumo. Fonte: consumo de alumínio primário - Metallgesellschaft (vários números); PIB = valores constantes (1980) expressos em milhões de dólares americanos: Economic Survey of Latin America and Caribbean. ECLA/UN (vários); Statistical Yearbook far Latin America. ECLA/UN (vários); IMF (1988); UI = expresso em toneladas/milhões de dólares americano PIB (1980) figure 3 - Comparing actual and estimated aluminium consumption and intensity of use based upon IU as a linear model of per capita GDP. A. Actual and fitted values for intensity of use; B. Actual and fitted values for consumption 
EXTENSÃ O DO MODELO TRANSLOG PARA IU E CONSUMO Partindo da premissa que o modelo lognormal empírico de Malenbaum possui uma base teó rica aceitável para relacionar IU e PEB per capita (y), pode-se obter, após sucessivas transformações matemáticas, as seguintes expressõ es para IU e consumo:

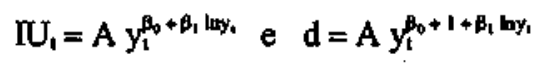

\section{De maneira análoga, tem-se que:}

$D_{1}=K Y_{1}^{a_{1}+a_{1}} m Y_{4}$

onde:

$\mathrm{D}_{4}=\mathrm{d}_{1}, \mathrm{POP}_{1} \quad$ e $\mathrm{Y}_{\mathrm{t}}=\mathrm{y}_{1}, \mathrm{POP}_{\mathrm{t}} \quad \mathrm{POP}_{4}=$ populaçăo.

O modelo de demanda derivada simplificado estabelece que a demanda é uma função do preço do produto $\left(\mathrm{P}_{\mathrm{t}}\right)$, do preço do substituto $\left(\mathrm{PS}_{\mathrm{t}}\right)$ e da renda $\mathrm{Y}_{\mathrm{t}}$. Quando este modelo é empregado para descrever o consumo através do tempo, deve ser incluída a mudança tecnológica $-c_{t}$. Assim, a função translog terá a seguinte forma:

$\mathrm{D}_{\mathrm{t}}=\mathrm{T}\left(\mathrm{Y}_{\mathrm{t}}, \mathrm{P}_{\mathrm{t}}, \mathrm{PS}_{\mathrm{t}} \tau_{\mathrm{t}} ; \Omega\right)$,

onde $\Omega$. é $O$ vetor dos parâmetros da função. De maneira análoga, tem-se: $\mathrm{D}_{\mathrm{t}} / \mathrm{Y}_{\mathrm{t}},=\mathrm{IU}_{\mathrm{t}}$, e $\mathrm{IU}_{\mathrm{t}}=\mathrm{T}\left(\mathrm{Y}_{\mathrm{t}}, \mathrm{P}_{\mathrm{t}}, \mathrm{PS}_{\mathrm{t}}, \tau_{\mathrm{t}} ; \Omega\right)$, onde $\Omega^{\prime}$ é idêntico a $\Omega$ exceto para o expoente de y pelo fator 1.0. Utiliza-se usualmente a regressão passo-a-passo (stepwise) para eliminar os produtos cruzados das variáveis redundantes e para atingir um modelo simplificado e de fácil representação.

Infelizmente, nenhum desses modelos pode ser aplicado diretamente para o consumo de alumínio no Brasil, pela dificuldade de se obter medidas adequadas de produtividade setoriais ou por mudança tecnológica no período analisado.

Deve-se ainda mencionar a dificuldade de utilizar os preços internacionais do metal (LME), devido a sucessivas intervenções do governo na regulamentação dos preços nacionais, principalmente após 1979. Para contornar esses problemas, a variável tempo foi utilizada como aproximação (proxy) para as mudanças tecnológicas e os preços foram corrigidos através de variáveis dummy.

Essa nova variável-preço $\left(\mathrm{Pw}_{\mathrm{t}}\right)$ ponderada baseia-se na observação de que até 1980 os preços domésticos obedeciam a tendência internacional, flutuando a seguir, o que resulta na seguinte" expressão calculada por Suslick \& Harris (1990b):

$$
\begin{aligned}
\operatorname{lnPw}_{\mathrm{t}}= & -7,6227+\operatorname{InPal},\left(2,0472 \cdot \mathrm{x}_{1 \mathrm{t}}+2,1057 \cdot \mathrm{x}_{2 \mathrm{t}}\right) \\
& -\operatorname{lnPa}_{\mathrm{t}} \cdot \operatorname{UnPcu}_{\mathrm{t}}\left(0,1365 \cdot \mathrm{X}_{1 \mathrm{t}}+0,1442 \cdot \mathrm{x}_{2 \mathrm{t}}\right)
\end{aligned}
$$

onde:

$\mathrm{Pal}_{\mathrm{t}}=$ preço internacional do alumínio $(\mathrm{LME})^{(2)}$

$\mathrm{Pcu}_{\mathrm{t}}=$ preço internacional do cobre $(\mathrm{LME})^{(2)}$

$\mathrm{x}_{1 \mathrm{t}}=1,1950 \leq \mathrm{t} \leq 1979$ e $\mathrm{x}_{1 \mathrm{t}}=0,1979<\mathrm{t} \leq 1987$

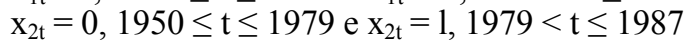

Com a regressão passo-a-passo (stepwise), obteve-se o seguinte modelo translog de consumo para o período 1950-1980:

$$
\begin{aligned}
& D_{1}=4,5277 \times 10^{-6} Y_{t}^{1,3587} \mathrm{Pw}_{1}^{-0,0824} \tau_{t}^{0,3339} \\
& R^{2}=0,971 \quad F(3,30)=343,5
\end{aligned}
$$

onde:

$\mathrm{D}_{\mathrm{t}}=$ consumo de alumínio

$\mathrm{Y}_{\mathrm{t}}=$ produto interno bruto - PIB (bilhões de dólares constantes referentes a 1980);

$\tau_{\mathrm{t}}=$ tempo com aproximação para a mudança tecnológica; $\mathrm{PW}_{\mathrm{t}}=$ variável-preço.
Nesse caso especial, o modelo translog se transforma num simples modelo linear logarítmico. Na equação acima, podese observar 0 grande efeito da variá vel (tempo) tecnologia e a pequena elasticidade do preço $(0,08)$. A figura 4 indica 0 ajuste do modelo translog no período 1950-1980 e os resultados da previsão para os anos 1981-1987. Embora o modelo exiba um bom ajuste para o período base, os resultados da projeção entre 1981-1983 não são muito expressivos.

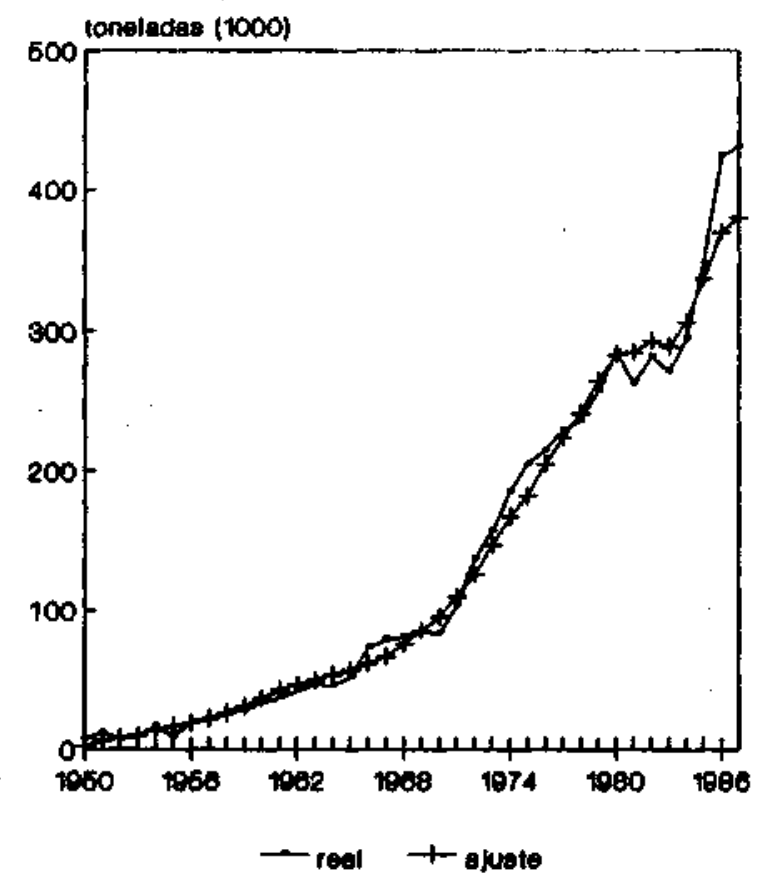

Figura 4 - Consumo de alumínio real e estimado baseado na extensão do modelo translog. Fonte: consumo de alumínio primário - Metallgesellschaft (vários números); $\mathrm{PIB}=$ valores constantes (1980) expressos em milhões de dólares americanos; Economic Survey of Latin America and Caribbean. ECLA/UN (vários); Statistical Yearbook for Latin America. ECLA/UN (varios);IMF (1988); IU = expresso em toneladas/milhões de dólares americano PIB (1980)

Figure 4 - Actual and estimated aluminium consumption based upon extensive translog model

MODELOS DE CONHECIMENTO DE IU Os modelos de conhecimento ou aprendizagem baseiam-se na noção intuitiva de que a performance de um sistema que é repetivivo e previsível pode ser resultado da aprendizagem. Essa técnica descreve a relação empírica entre as quantidades produzidas (output) e certas quantidades de entrada (input), nas quais o aumento de produtividade, fruto do conhecimento, é observável.

Esses modelos são muito utilizados como instrumentos de planejamento nas áreas de avaliação e previsão da produtividade, avaliação de custos e análises econômicas em geral (Belkaouni 1986). Exemplos dos modelos de conhecimento no planejamento mineiro e plantas metalúrgicas podem ser encontrados em Pierson (1981).

Segundo Suslick \& Harris (1990), existem duas motivações básicas para o emprego dos modelos de conhecimento na previsão do consumo mineral. A primeira motivação baseia-se nas mudanças tecnológicas provocadas na utilização dos materiais que refletem um certo grau de aprendizado. A outra 
motivação está ligada à natureza das séries temporais, na qual existe uma forte analogia entre essas e as derivadas do tempo que são muito mais susceptíveis para a modelizacão.

Em termos formais, o modelo de conhecimento pode ser definido por meio das seguintes relações, que representam simples valores acumulados:

$$
\frac{\dot{D}_{\mathrm{t}}}{\overline{\mathrm{Y}}_{\mathrm{t}}}=\mathrm{L}\left(\dot{\mathrm{Y}}_{\mathrm{t}}\right)
$$

onde:

$\stackrel{*}{n}=$ consumo mineral acumulado

$\stackrel{*}{\mathrm{Y}},=$ PEB per capita acumulado; e

$\mathrm{L}(\mathrm{)}$ ) função de conhecimento ou aprendizado.

Assim que os parâmetros do modelo de conhecimento $\mathrm{L}()$ para ${ }_{\mathrm{W}}^{*}$ são determinados, o modelo pode ser transformado para descrever o consumo anual, $\mathrm{D}_{\mathrm{t}}$ :

$\mathrm{D},=\left[\mathrm{L}^{\prime}\left(\stackrel{*}{\mathrm{Y}_{\mathrm{t}}}\right) \stackrel{*}{\mathrm{Y}} \mathrm{t},+\mathrm{L}\left(\stackrel{*}{\mathrm{Y}_{\mathrm{t}}}\right)\right] \mathrm{Y}_{\mathrm{t}}$, onde

$L^{\prime}\left(Y_{1}\right)=\frac{d\left[W_{1}\right]}{d t} \times \frac{d\left[L\left(\bar{Y}_{1}\right)\right]}{d t}$

$Y_{1}=\frac{d\left[Y_{1}\right]}{d t}$

A figura 5A mostra um ajuste de $\mathrm{W}$, em relação a $\mathrm{Y}$,. Um padrão robusto pode ser obtido pela eliminação dos cinco pontos iniciais, e a aplicação da regressão linear simples produz a seguinte equação:

$$
\ln \stackrel{*}{W}=-10,6487+0,7763 \ln \dot{*}_{1} \quad R^{2}=0,995
$$

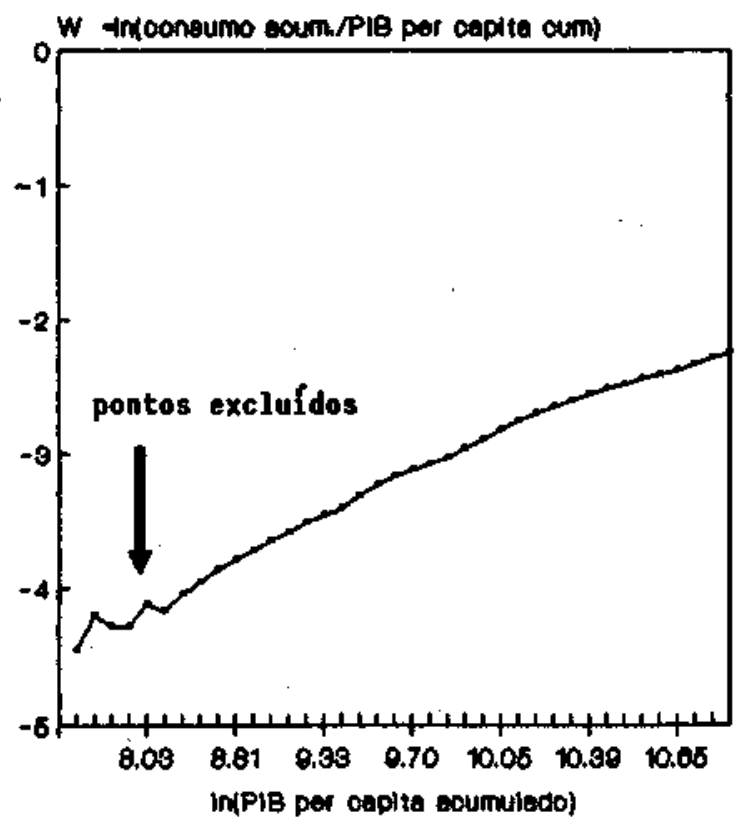

onde:

$\tilde{\mathrm{W}}_{\mathrm{t}}{ }^{\mathrm{w}}$ consumo acumulado/PIB per capita acumulado $\stackrel{*}{\mathrm{Y}}_{\mathrm{t}}=$ PIB per capita acımulado.

De maneira análoga, $\mathrm{W}_{\mathrm{t}}=2,373 \times 10^{-5} \mathrm{Y}_{\mathrm{t}} \mathrm{e}$, segundo a equação (9),

$$
\mathrm{D},=4.2147 \times 10-{ }^{-} \mathrm{Y}_{\mathrm{t}} \cdot{ }_{\mathrm{Y}}^{*}{ }_{\mathrm{t}}^{0,7631}
$$

A figura 5B mostra o resultado do modelo de conhecimento simples para o alumínio, no qual o consumo para os anos futuros é estimado pela projeção do PIB per capita.

Segundo Harris (1 984), o sistema de conhecimento simples pode ser transformado numa forma mais complexa, que permite descrever as variáveis intervenientes no consumo mineral de maneira mais abrangente:

$\tilde{Z}_{1}=\mathrm{T}\left(\dot{Y}_{1}, \mathbb{P W}_{\mathrm{L}}, \tilde{I}_{1}\right)$

onde:

$$
\dot{z_{i}}=\dot{V}_{1} / \dot{Y}_{\mathfrak{r}}
$$

$$
\begin{aligned}
& \dot{V}_{t}=\sum_{i}^{t} D_{i} \cdot P_{W_{i}} \quad \text { valor acumulado } \\
& \dot{Y}_{t}=\sum_{i}^{1} Y_{i} \quad \text { renda per capita acumulada } \\
& \dot{P}_{W_{1}}=\sum_{i}^{1} P w_{i} \quad \text { preco ponderado acumulado } \\
& \dot{\Gamma}_{t}=\sum_{i}^{1} \Gamma_{i} \quad \text { fator tecnológico acumulado }
\end{aligned}
$$

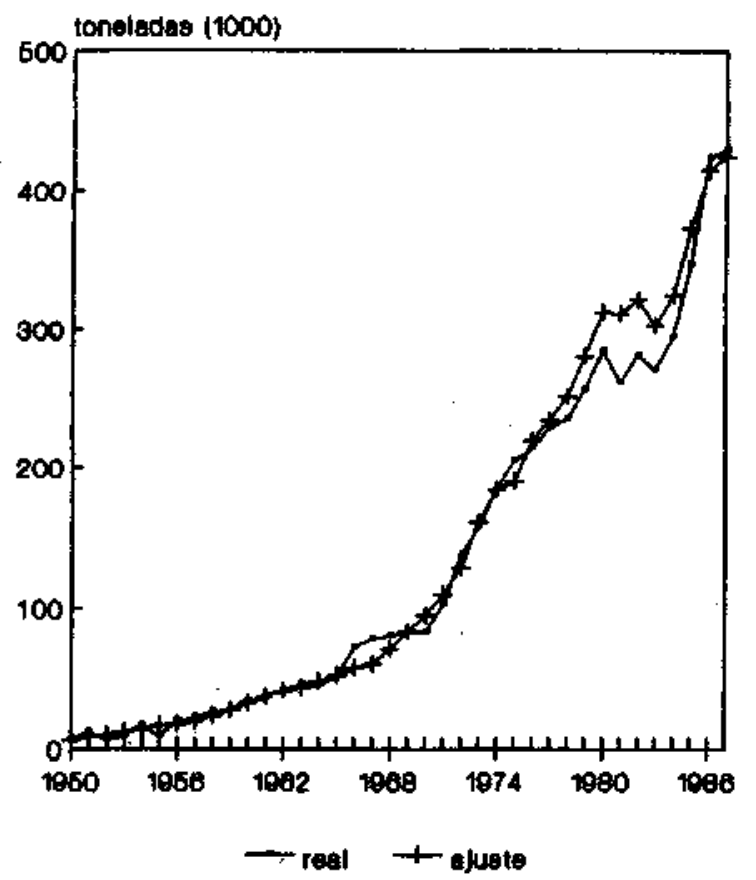

Figura 5 - Previsão baseada no sistema de conhecimento simples. $\boldsymbol{A}$. Relação de conhecimento simples; $\boldsymbol{B}$. Consumo real e estimado no modelo de conhecimento simples. Fonte: consumo de alumínio primário - Metallgesellschaft (vários números); $\mathrm{PIB}=$ valores constantes (1980) expressos em milhões de dólares americanos; Economic Survey of Latin America and Caribbean. ECLA/UN (vários); Statistical Yearbook for Latin America. ECLA/UN (vários); IMF (1988); IU=expresso em toneladas/milhões de dólares americano PIB (1980)

Figure 5 - Forecasting based upon simple learning model. A. The simple learning relation; B. Actual and estimated consumption based upon the learning model 
O consumo de alumínio, por esse modelo de conhecimento modificado, pode ser visualizado na figura 6 , cuja equação final, após diversas transformações, é a seguinte:

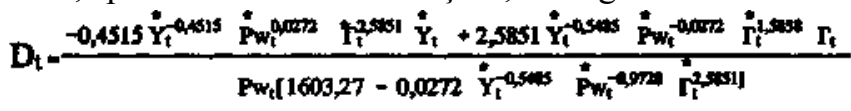

toneidedes (1000)

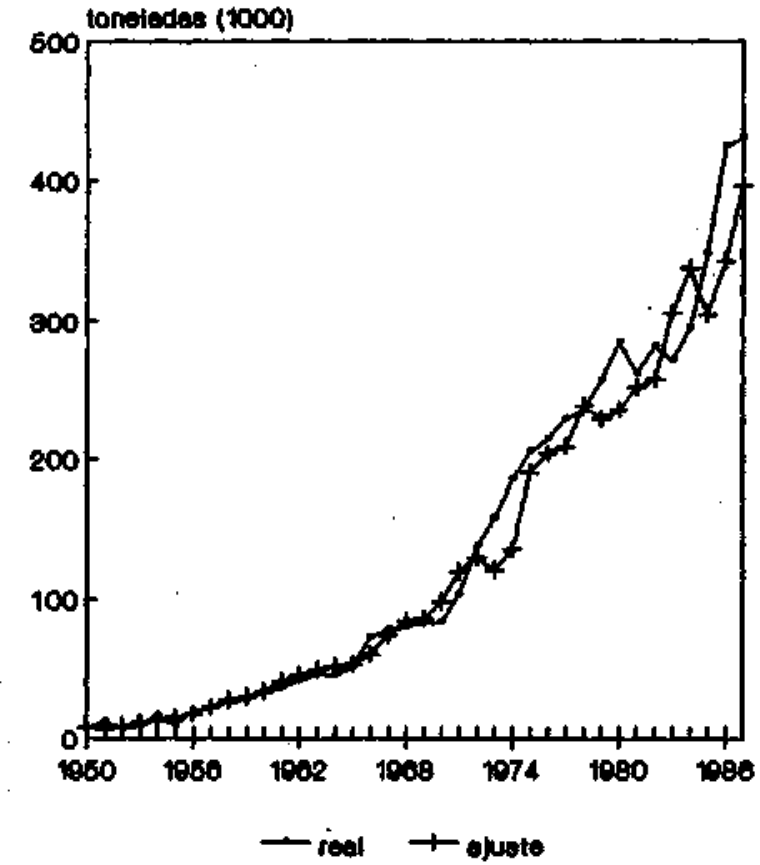

Figura 6 - Consumo real e estimado baseado no modelo de conhecimento modificado. Fonte: consumo de alumínio primário - Metallgesellschaft (vários números); PIB - valores constantes (1980) expressos em milhões de dólares americanos; Economic Survey of Latin America and Caribbean. ECLA/UN (vários); Statistical Yearbook for Latin America. ECLA/UN (vários); IMF (1988); IU - expresso em toneladas/milhões de dólares americanos PIB (1980) Figure 6 - Actual and estimated consumption based upon the modified learnig model'

\section{PROJEÇÃO DE VETORES MACROECONÔMICOS}

Um dos aspectos essenciais da economia é a inter-relação de suas variáveis, que nem sempre é levada em consideração pelos métodos econométricos usuais, principalmente quando algumas variáveis econômicas apresentam certa correlação.

Uma alternativa para superar essas limitações é substituir as $m$ variáveis explanatórias por $m$ novas variáveis, denominadas escores fatoriais, que são linearmente independentes. A vantagem desta técnica é a de respeitar a correlação entre as variáveis sem provocar distorções, e incrementar sensivelmente as previsões (Harris 1989).

No caso do consumo de alumínio, que consiste de duas variáveis explanatórias, $\mathrm{Y}_{\mathrm{t}}$ (renda per capita) e $\mathrm{Pw}_{\mathrm{t}}$, (variáveis-preço construídas para o alumínio no Brasil), podem ser obtidas por análise fatorial, equações para os escores fatorias (Fã e F\&) com comunalidade igual a 1,0, que são utilizados na equação do consumo:

$\mathrm{D},=-125,924689+10,3038 \mathrm{~F}_{\mathrm{h}}+32,6694 \mathrm{~F} 2$.

Segundo Harris (1989), este modelo pode ainda ser transformado na condição especial de coeficientes temporais, calculando-se $\mathrm{AD}, \mathrm{ADt} / \mathrm{AFu}$, e $\mathrm{ADj} / \mathrm{AF}^{*}$ para cada observação, que fornecem ajustes mais robustos. Quando um ou mais gráficos exibem uma tendência expressiva, trata-se de uma indicação para caso especial de coeficientes variados no tempo. Flutuações em tomo de um valor médio recomendam o uso do caso clássico dos coeficientes fixos.

$\mathrm{O}$ exame das tendências dos coeficientes do modelo fatorial para o consumo de alumínio no Brasil denota a presença de fortes trends, indicando a necessidade do emprego desses coeficientes temporais para o exercício da previsão. A equação da função temporal dos coeficientes é a seguinte:

$$
D_{t}=I, 0488 f_{0}(t)+0,9787 f i(t) F i(t)+I, 0180 f_{2}(t) F_{2}(t)
$$

A figura 7 mostra o ajuste do modelo vetorial; a previsão do consumo de alumínio para o ano 2000 foi calculado com projeção dos coeficientes e dos escores fatoriais linearmente em relação ao tempo.

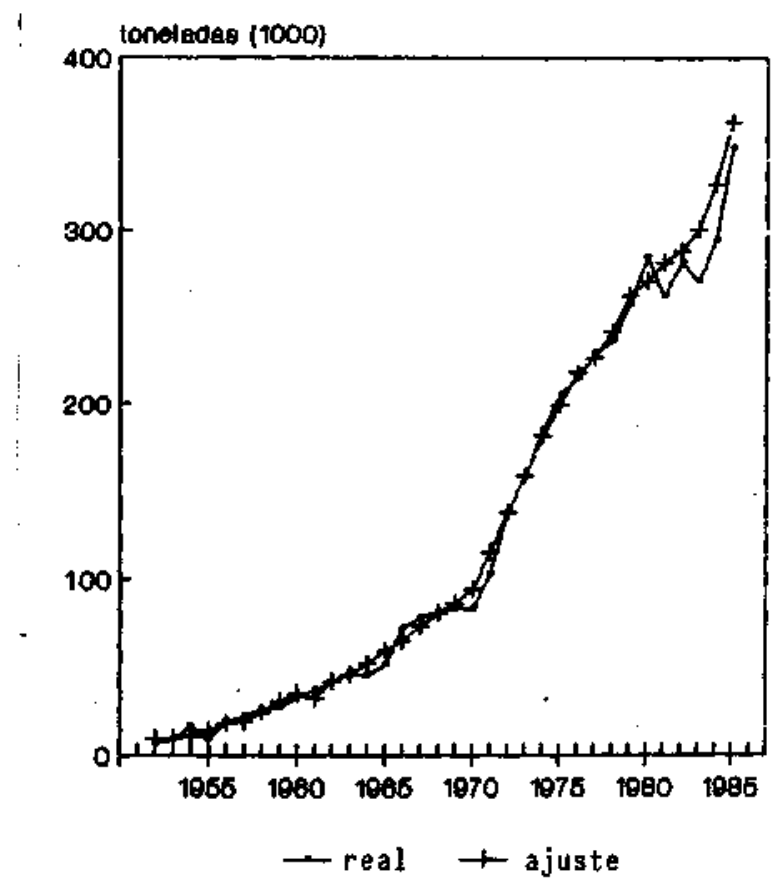

Figura 7 - Consumo real e estimado baseado no modelo de coeficiente temporal (modelo fatorial); Fonte: consumo de alumínio primário - Metallgesellschaft (vários números); PIB - valores constantes (1980) expressos em milhões de dólares americanos; Economic Survey of Latin America and Caribbean. ECLA/UN (vários); Statistical Yearboor for Latin America. ECLA/UN (vários); IMF (1988); IU - expresso em toneladas/milhões de dólares americanos PIB (1980)

Figure 7 - Actual and estimated consumption based upon the time varying coefficient (factor model)

AVALIAÇÃO DOS MÉTODOS UTILIZADOS Um dps aspectos mais importantes na avaliação de qualquer previsão é a capacidade do método empregado reproduzir as condições reais do processo da demanda mineral. Esta restrição obriga o uso de critérios de validação, que consistem em testes para avaliar se o método é uma representação adequada dos elementos e das relações do sistema em referência.

De modo geral, os elementos essenciais que devem compor o processo de validação podem ser resumidos nos seguintes: - significado estatístico dos parâmetros configurados no modelo;

- correspondência entre os valores estimados ("ex-ante e ex-post") pelo método proposto e os valores reais ou medidos; - sensibilidade do método em identificar possíveis mudanças no ritmo do consumo. 
Neste trabalho será utilizado como critério de exatidão para avaliar a "performance" dos valores simulados, o erro médio absoluto (AAE), definido pela seguinte expressão:

$$
\mathrm{AAE}-\sum_{\mathrm{i}=1}^{n}\left(\frac{\mathrm{Rc}-\mathrm{Pr}}{\mathrm{Rc}}\right) \text { i } / \mathrm{n}
$$

onde:

Rc - valor real ou observado;

$\mathrm{Pr}$ - valor previsto ou simulado pelo método de previsão e $\mathrm{n}$ - intervalo de tempo.

A ayâUaçiio dos resultados foi realizada em dois níveis. No prímejrp, cobrindo o período-teste (1981-1987) os dados de consumo medido foram eliminados para a estimativa dos modelos. No passo seguinte, o conjunto global de dados (1950-1987) foi considerado na avaliação e, em seguida, utilizado para a projeção do consumo no ano 2000. Os resultados dessas simulações estão indicados na tabela 2.

Tabela 2 - Síntese dos resultados obtidos pelos diferentes métodos na previsão do consumo de alumínio, com base no cálculo do erro médio absoluto (AAE)

Table 2 - Results of different methods for aluminium consumption forecasting based upon average absolute errors (AEE)

\begin{tabular}{|c|c|c|c|c|}
\hline Matodo & $\begin{array}{c}\text { Periodo } \\
\text { Observedo }\end{array}$ & AAP & $\begin{array}{l}\text { Pedodo } \\
\text { Exposil }\end{array}$ & AAE \\
\hline Thend Linear IU* & $1950-80$ & 0,12 & $1981-87$ & 0,10 \\
\hline Modelo Lhear IJ*" & $1950-80$ & 0,22 & 1981.87 & 0,13 \\
\hline Modelo Lhent IJ"x & $1950-87$ & 0,21 & & \\
\hline Modelo Lagmomil I0 & $1950-10$ & 0,12 & $1981-87$ & 0,12 \\
\hline Madelo Lopiomal IU & $1950-87$ & 0,12 & & \\
\hline Eavendo Thuslas Coos. & $1950-80$ & 0,13 & $1981-87$ & 0,09 \\
\hline Extunito Thesiby Cons. & $1950-87$ & 0,11 & & \\
\hline Madelo Conhecimento IIJ & $1950-80$ & 0,15 & 1981-87 & 0,07 \\
\hline Modtu Ẹit. Crihedimento & $1980-100$ & 0,14 & 1981-87 & 0,11 \\
\hline 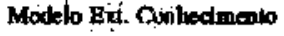 & $1950-87$ & 0,14 & & \\
\hline Modelo Cod. Tetupanin & $1052-80$ & 0,10 & $1991-85$ & 0,07 \\
\hline Madslo Cod. Tepponds & $1952-85$ & 0,09 & & \\
\hline
\end{tabular}

A avaliação do período ex- "post" (1981-1987) mostra que os métodos de curva de conhecimento e dos coeficientes temporais são os que apresentam os melhores valores de exatidão $(0,07)$. $\mathrm{O}$ modelo translog é ligeiramente inferior $(0,09)$. Os modelos com valores mais baixos de exatidão são IU linear, seguidos do lognormal e de conhecimento modificado em ordem decrescente.

Em relação à descrição do consumo de alumínio no período 1950-1987, os métodos lognormal, translog e de coeficientes temporais exibem os melhores ajustes. Para os métodos lognormal e translog, este comportamento é devido à forte componente associada ao PIB, confirmando os resultados encontrados por Suslick \& Harris (1990).

A tabela 3 sintetiza os resultados obtidos com a projeção do consumo de alumínio para o ano 2000, utilizando as equações do período 1950-87. As projeções basearam-se nos pressupostos de que os preços de alumínio e cobre se manterão dentro dos patamares atuais de década. As estimativas do PIB basearam-se em dois cenários com crescimento médio anual de 3,6\% e 6,1\%, segundo o World Bank (1988), fornecendo dois níveis, inferior e superior, respectivamente.

A amplitude das previsões apresenta-se bastante variada, mas, pôr outro lado, pode-se assumir o intervalo entre 800.000 a 1.300.000 toneladas métricas, que inclui as previsões dos modelos, translog e de coeficientes temporais com melhores valores de exatidão no período ex-"post".
Tabela 3 - Projeção do consumo de alumínio no Brasil para o ano 2000 gerada pelos diferentes métodos

Table 3 - The year 2000 Brazil demand forecasts for aluminium consumption by different methods

\begin{tabular}{|c|c|c|c|}
\hline Matado & \multicolumn{2}{|c|}{ Conguno de Ahminio" } & Stperia \\
\hline Trend Linto IU & 15118 & NA & NA \\
\hline Modelo Linter IU & NA & 983,8 & 1752,8 \\
\hline Modelo Loganall IU & NA & 531,0 & 6045 \\
\hline Extensio Thalog Contumo & NA & 829,1 & 1249,8 \\
\hline Madelo Conhecinendo IU & NA & 10909 & 1588,7 \\
\hline Extento Conbecimento IU & MA & 8620 & 997,7 \\
\hline Cot. Tompanis Conguno & NA & 1009,1 & 1379,4 \\
\hline
\end{tabular}

$(*)$ Valores expressos em 1.000 toneladas métricas.

TEMPO - método de previsão baseado unicamente na projeção em função do tempo. PIB e PIB per capita obtidos de previsão do WORLD BANK (1988)

Limite inferior - taxa média anuuual de crescimento do PIB estimada on 3,6\% no período 1987-2000.

Limite superior - taxa média anual de crescimento do PIB estimada on $6,1 \%$ no periodo $1987-2000$

NA • não se aplica.

Este intervalo aproxima-se das previsões realizadas pelo World Bank (1983b), coincidindo com o modelo translog de consumo. Esse modelo inclui, entre as suas variáveis, o preço, o PIB e o tempo, como aproximação para mudança tecnológica, que representam todos os fatores interyenientes no consumo de alumínio ao longo do tempo: atividade econômica, substituição, preço e mudança na composição e na tecnologia dos produtos de alumínio.

Um outro aspecto importante na avaliação dos métodos de previsão é a relação entre o custo e a exatidão. Diversos estudos realizados sobre esses dois parâmetros mostram que a decisão de incrementar a exatidão implica sempre em custos proporcionalmente crescentes. No contexto da previsão mineral, esta relação nem sempre é direta, ou seja, a simples utilização de um método mais complexo não implica automaticamente em ganhos consideráveis na exatidão. Outros parâmetros devem participar na avaliação, tais como: pré-requisitos e qualidade das informações, natureza das técnicas estatísticas de validação, facilidade de manuseio e compreensão por indivíduos não diretamente envolvidos no processo de previsão, dentre outros parâmetros.

Por causa desses aspectos foi estabelecido um esquema qualitativo que abrange todos os fatores considerados essenciais na previsão: exatidão, custo., complexidade, identificação das mudanças tecnológicas e pré-requisitos do método (característica da informação etc.).

O parâmetro custo se refere a diferentes categorias (construção do modelo, manutenção e utilização do modelo, volume de cálculo etc.). A complexidade mede a transparência que as relações entre as variáveis e a estrutura do modelo possibilitam para a compreensão para indivíduos não familiarizados com as técnicas quantitativas de previsão.

A identificação das mudanças tecnológicas se exprime pela sensibilidade do modelo aos efeitos de substituição dos materiais e transformações provocadas pelo desenvolvimento tecnológico. O fator pré-requisito envolve: a disponibilidade de séries históricas, nível de agregação, grau de conhecimento dos operadores e período necessário para a execução da previsão.

Estabeleceu-se uma escala, arbitraria entre alta, média e baixa para a "performance" dos métodos em relação aos fatores, conforme mostra a tabela 4. Apesar desta avaliação ser essencialmente subjetiva na atribuição dos peso, pode-se observar que não existe um único método que atenda integralmente às exigências de todos os fatores.

Uma outra forma de representação pode ser visualizada na figura 8 , onde interagem as ponderações entre complexidade, exatidão e custo, em parte refletidas na curva de custo de previsão, na qual os métodos mais simples carecem de exatidão compatível. Pode-se observar nesse gráfico que a deci- 
Tabela 4 - Avaliação qualitativa dos métodos de previsão Table 4 - Qualitative evaluation of forecasting methods

\begin{tabular}{|c|c|c|c|c|c|c|}
\hline \multirow[t]{2}{*}{ Medodo } & \multicolumn{2}{|c|}{ Extdids } & \multirow[t]{2}{*}{ Consos } & \multirow[t]{2}{*}{ Condilariduis } & \multirow[t]{2}{*}{ Toceloda } & \multirow[t]{2}{*}{ Rentititos } \\
\hline & $1960-47$ & 1981-87 & & & & \\
\hline Thand Lech (Tapo) & mbitis & netilu & bino & beteo & bixo & betixo \\
\hline nod Liner (AB) & bum & nolit & biso & binos & Wito & bixiog \\
\hline 1epond & matis & ridale & wiso & $b=0$ & miro & tetso \\
\hline 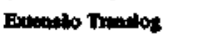 & stith & ulu & thedito & mito & ath & atsolb \\
\hline Contuctuma I & $b+x$ & alta & $b+9$ & bino & Lillo & bixo \\
\hline 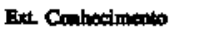 & buin & 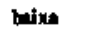 & mótio & molis & motio & modis \\
\hline Oof: Temponila & sthe & 背战 & 16 & هإ & a & madio \\
\hline
\end{tabular}

Obs- exatidão: alta significa exatidão excelente, isto é, valores baixos de AAE complexidade: alta implica nun nível elevado de complexidade e baixa transparência $\mathrm{p} /$ usuários.

tecnologia: alto significa que o método incorpora os efeitos da mudança tecnológica. requisitos: alto implica que para a execução do modelo o número de requisitos é elevado.

CUSTOS CRESCENTES

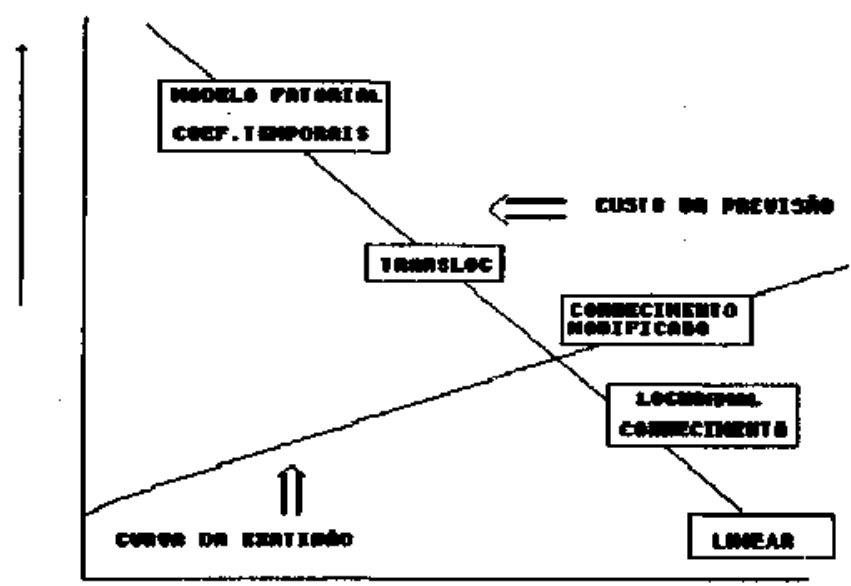

Dininutcho do complexidade exatids

Figura 8 - Avaliação qualitativa dos métodos de previsão de complexidade, exatidão e custo

Figure 8 - Qualitative evaluation of forecasting methods based upon complexity, accuracy, and cost

são de incrementar a exatidão implica sempre em custos proporcionalmente crescentes, embora na previsão mineral esta relação nem sempre seja direta, isto é, a simples utilização de um método mais complexo não implica ganhos consideráveis na exatidão.

CONSIDERAÇÕES FINAIS A previsão da demanda numeral possibilita o reconhecimento e a análise futura do comportamento de variáveis econômicas, que constituem instrumentos essenciais no auxílio ao planejamento das atividades privadas e de organismos governamentais do setor mineral.

Este trabalho visou realizar a previsão do consumo de alumínio primário no ano 2000 , baseando-se em modelos econômicos, métodos quantitativos e técnicas direcionadas para a projeção de longo prazo. Apesar de cobrirem um amplo espectro de aplicações e de fornecerem ajustes adequados em casos específicos, constata-se que os modelos, isoladamente, não permitem preencher todos os requisitos exigidos na previsão, tais como definição do consumo, qualidade dos dados, substituição, mudança tecnológica, preços e custos.

A análise dos resultados da exatidão (erro médio absoluto) no período teste entre 1981 e 1987 indica que os métodos de conhecimento simples e de coeficientes temporais seguidos pelo modelo translog exibem os melhores valores de exatidão. $\mathrm{O}$ modelo de $1 \mathrm{U}$ linear apresenta valores baixos de exatidão, seguido pelo modelo lognormal e de conhecimento modificado.

Tomando-se como referências os diferentes resultados de exatidão e os padrões de comportamento dos ajustes dos modelo, o consumo de alumínio no Brasil para o ano 2000 deve situar-se entre 800.000 a 1.300 .000 toneladas métricas. Este intervalo abrange a previsão do modelo translog e do coeficiente temporal que forneram altos valores de exatidão no período teste (1981-1987).

O limite inferior desta previsão está associado a taxas baixas de crescimento anual do PTB (em tomo de $3 \%$ ), enquanto o limite superior relaciona-se a uma taxa anual de crescimento de $6 \%$. Na hipótese pessimista de um nível de crescimento moderado até o final da década, o consumo de alumínio praticamente será o dobro do constatado durante 1986.

Agradecimentos O autor agradece ao professor DeVerle P. Harris da University of Arizona, que proporcionou todas as condições para o desenvolvimento deste trabalho, aos professores Iran F. Machado e Luiz A. Milani, pela leitura e sugestões ao manuscrito, e à Fundação de Amparo à Pesquisa do Estado de São Paulo (FAPESP) pelo apoio financeiro durante a sua permanência Fundação de Amparo à Pesquisa do Estado de São Paulo na University of Arizona durante o ano sabático de 1989.

\section{REFERÊNCIAS BIBLIOGRÁFICAS}

ASSOCIAÇÃO BRASILEIRA DE ALUMÍNIO 1988. Anuário Estatístico. São Paulo, ABAL. 15 p.

ALRAWAffl, K. \& RUBBER, M. 1990. Embodied net exports, the effect on intensity of use estimation in the mineral demand forecasting; U.S. Sulfur 1965-1985. Resources Policy, 16(3):200-208.

ARANHA, A.C. 1988. A Indústria do Aluminio: Um Histórico e Perspectiva para os Anos 90. Campinas. IG/UNICAMP. 16 p. (Conferência).

BELKAOUNI, A. 1986. The Learning Curve: A Management Accounting Tool. London, London Quorum Books. $245 \mathrm{p}$.

BRAZ-PEREIRA, E. 1988. Brazil: The Transition to An Export Industry. In: PECK, M.J. ed. The World Aluminum Industry in a Changing Energy Era. Washington, Resource for the Future, p. 148-174.

CANAVAN, P.O. 1981. The Determination oflntensity-of-Use: A Case Study of Tin Solder end Use. Pennsylvania. 197 p. (PhD Dissertation, University Park/University State University).

CONSIDINE, T.J. 1991. Economic and technologic determinants of materials intensity of use. Land Economic, 67(1):99-115.

ECONOMIC COMISSION FOR LATIN AMERICA AND THE CARIBBEAN (vários anos). Economic Survey of Latin America and Caribbean. New York, ECLA/UN.

ECONOMIC COMMISSION FOR LATIN AMERICA AND THE CARIBBEAN (vários números). Statistical Yearbook for Latin America. New York, ECLA/UN.
GREGG, J.V.; HOSSELL, C.H.; RICHARSON, J.T. 1964. Mathematical trend curves: an aid to forecasting. Mathematical and Statistical Techniques for Industry. Toronto, D. Van Mostrand. 100 p. (Monograph 1).

HARRIS, D.P. \& JEOM, G.J. 1987. Improved Methods for Long-Range Forecasting. Tucson, Mineral Economics/Department of Mining and Geological Engineering/College of Engineering and Mines/University of Arizona. 161 p. (Research Project)

.HARRIS, D.P. 1984. Mineral Resources Appraisal - Mineral Endowment, Resources and Potential Supply: Concepts, Methods and Cases. Oxford, Claredon Press. 445 p.

HARRIS, D.P. 1989. Forecasting of Mineral Industries. Tucson, Mineral Economics Program/Department of Mining and Geological Engineering/College of Mines/University of Arizona. 220 p. (Lectures notes MnEc 665).

HUTCHINSON, R.S. \& TILTON, J.E. 1987. Is the intensity of copper use still declining in the USA? Natural Resources Forum, 11(4):325-334.

INTERNATIONAL MONETARY FUND 1988. Yearbook 88. International Finance Statistics. Washington, IMF. 82 p.

MALENBAUM, W. 1973. Material Requirements in the United States and Abroad in the Year 2000. Philadelphia, University of Pennsylvania Press. (Research project prepared for the National Commission on Materials Policy). 
MALENBAUM.W. 1975. Law of demand for minerals, In: Amual Meeting of the American Institute of Mining, Metallurgical and Petroleum Engineers, 104 . Proceedings of the Council of Economic, p. 147-155.

MALENBAUM, W. 1978. World Demand for Raw Materials in 1985 and 2000. New York, MacGraw Hill. 126 p.

METALLGESELLSCHAFT AKTTENGESELLSCHAFT (vários números). Metal statistics. Frankfurt-am-Main, Metallgesellschaft Ag.

PERSON, O. 1981. Learning curves make productivity gains predictable. Eng- Mining Journal, (8):56-64.

ROBERTS, M.C. 1985. Theory and Practice of the Intensity of Use Method of Mineral Consumptiom Forecasting. Tucson. 350 p. (PhD Dissertation, Mineral Economics/University of Arizona).

ROBERTS, M.C. 1988. What caused the slack demand for metals after 1974 ? Resources Policy, 14(3):231-246.

SUSLJCK, S.B. 1990. Métodos de Previsão da Demanda Mineral. Campinas. 150 p. (Tese de Livre-Docência, IG/UNICAMP).

SUSLICK, S.B. \& HARRIS, D.P. 19906. Latin American metal consumptionrecent trends and determinants. Materials \& Society. 16(3): 184-199.
SUSLICK, S.B. \& HARRIS, D.P. 1991. Long-range consumption forecasts using innovative methods: the case of aluminum in Brazil to the Year 2000. Resources policy, 15(4):351-372.

TTLTON, JJE. 1985. Atrophy in metal demand. Earth Min. Sci., 54(2): 15-18.

WORLD BANK 1983a Brazil: Industrial Policies and Manufactured Export. Washington, World Bank. 163 p. (A World Bank Country Study).

WORLD BANK 1983b. Worldwide Investment Analysis: The Case of Aluminum. Washington, World Bank Number 603, 269 p. (A World Bank Staff Working Paper).

WORLD BANK 1988. World Development Report 1988. New York, Oxford University Press.

MANUSCRITO A675

Recebido em 21 de setembro de 1990 Revisão do autor em 5 de fevereiro de 1991 Revisão aceita em 5 de fevereiro de 1991 